\title{
Carcinoma avanzado de conductos colectores. Reporte de tres casos
}

Almeida-Magaña R, Aguilar-Davidov B, Priego-Niño A, Castillo-Canto C

\section{Resumen}

ANTECEDENTES: El carcinoma de conductos colectores es una neoplasia de células renales, extremadamente rara, cuya incidencia varía de 0.5 a $3 \%$ de los tumores malignos del riñón. Es un tumor agresivo, de crecimiento local, rápidamente invasivo y con evolución a metástasis temprana.

CASO CLÍNICO: Se reportan tres casos de carcinoma de conductos colectores en etapas avanzadas confirmado mediante estudio histológico. Se revisaron las características clínicas de manera retrospectiva. Todos los pacientes tenían antecedente de tabaquismo, no manifestaban síntomas al momento del diagnóstico y se estadificaron en etapa clínica avanzada. Todos los pacientes fueron tratados con nefrectomía radical, fallecieron antes de cumplirse dos meses de la intervención quirúrgica: 2 por complicaciones posquirúrgicas y 1 por evolución temprana de la enfermedad.

CONCLUSIONES: La nefrectomía citorrreductora puede ser una fuente de morbilidad y mortalidad, se requieren series más amplias para definir la función del tratamiento del carcinoma de conductos colectores.

PALABRAS CLAVE: Carcinoma de conductos colectores; nefrectomía radical; cáncer renal; complicaciones posoperatorias.

Rev Mex Urol. 2018 Jan-Feb;78(1):67-72.

\section{Advanced collecting duct carcinoma in three patients}

Almeida-Magaña R, Aguilar-Davidov B, Priego-Niño A, Castillo-Canto C

\section{Abstract}

BACKGROUND: Collecting duct carcinoma is an extremely rare type of renal cell carcinoma and accounts for 0.5 to $3 \%$ of malignant kidney cancers. It is an aggressive, rapidly invasive disease that progresses to early metastasis.

CLINICAL CASE: Three cases of advanced-stage collecting duct carcinoma confirmed through histologic study are reported herein. The clinical characteristics of the patients were reviewed retrospectively. All the patients had a history of smoking, were symptomatic at the time
Servicio de Urología, Centro Médico Nacional Lic. Manuel Ávila Camacho (IMSS) Puebla, México.

Recibido: julio 2017

Aceptado: enero 2018

Correspondencia

Bernardo Aguilar Davidov

badavidov@hotmail.com

Este artículo debe citarse como

Almeida-Magaña R, Aguilar-Davidov B, Priego-Niño A, Castillo-Canto C. Carcinoma avanzado de conductos colectores. Reporte de tres casos. Rev Mex Urol. 2018 ene-feb;78(1):67-72.

DOI: https://doi.org/10.24245/revmexurol.v78i1.1541 
of diagnosis, and presented with advanced-stage disease. The three patients underwent radical nephrectomy and all died before the end of two postoperative months: 2 of the deaths were due to postoperative complications and 1 from early disease progression.

CONCLUSIONS: Cytoreductive nephrectomy may be a source of morbidity and mortality. Larger case series are required to define its role in the treatment of collecting duct carcinoma.

KEYWORDS: Collecting duct carcinoma; Radical nephrectomy; Kidney cancer; Postoperative complications.

\section{ANTECEDENTES}

El carcinoma de conductos colectores es una neoplasia de células renales que se origina, como su nombre lo indica, en las células principales de los conductos colectores. Esta enfermedad es extremadamente rara, pues representa de 0.5 a $3 \%$ de los tumores malignos del riñón. ${ }^{1}$ El carcinoma de conductos colectores es muy agresivo, de crecimiento local rápidamente invasivo y evolución a metástasis temprana. La mayoría de los pacientes suele diagnosticarse en etapas tardías. Aún se discute si el pronóstico es peor que el de pacientes con carcinoma de células claras, etapa por etapa. ${ }^{2}$

El carcinoma de conductos colectores crece de forma infiltrante, con preservación de la silueta renal e invasión frecuente a la grasa perinéfrica y perisinusal, así como a órganos vecinos. La cirugía radical es el tratamiento de elección, pero existe evidencia limitada acerca de su efectividad como tratamiento citorreductor. ${ }^{3}$

Puesto que se trata de un tumor maligno poco común, el diagnóstico diferencial es difícil de establecer. Eble y la OMS emitieron criterios diagnósticos que se utilizan en casi todos los estudios y recientemente se han revisado. ${ }^{4}$
Aunque el tratamiento antiangiogénico se ha prescrito en otros tipos de cáncer renal, en el carcinoma de conductos colectores ha demostrado poca efectividad. Diversos estudios han evaluado la eficacia de la quimioterapia, especialmente la combinación de gemcitabinacisplatino, que demostró reacción parcial en un grupo de pacientes y un beneficio potencial en cuanto a supervivencia. ${ }^{5}$

La mayor parte de las investigaciones asociadas con estos tumores incluye casos clínicos, con sólo 4 series grandes y 1 estudio prospectivo, recientemente publicados en una revisión sistemática. ${ }^{6,7}$

El objetivo de este artículo es reportar el curso clínico y las características histológicas de tres pacientes diagnosticados con carcinoma de conductos colectores en etapa avanzada.

\section{REVISIÓN DE LOS CASOS CLÍNICOS}

Se analizaron 345 reportes de patología de tumores renales, realizados en el servicio de Urología del Centro Médico Nacional Lic. Manuel Ávila Camacho (IMSS) de Puebla, entre enero de 2009 y mayo de 2015. Se encontraron 3 pacientes con diagnóstico final de carcinoma de conductos colectores. Todos los estudios fueron revisados 
en el departamento de Patología. Se evaluaron las variables demográficas y clínicas de edad, sexo, síntomas, comorbilidades, lado afectado, tratamiento, características radiológicas e histológicas, etapa clínica (criterios TNM de la American Joint Committee on Cancer de 2010), estado funcional (escala del Eastern Cooperative Oncology Group [ECOG]) y supervivencia posterior a la nefrectomía. Las características clínicas y patológicas se muestran en el Cuadro 1. Todos los pacientes fueron hombres, con edad media de 60.6 años al momento del diagnóstico; la evaluación radiográfica inicial mostró un tumor renal, sin realce con el medio de contraste, de crecimiento infiltrante (Figura 1) y adenomegalias retroperitoneales. Puesto que el estado funcional inicial de los pacientes era adecuado, se decidió efectuar nefrectomía radical de alta complejidad técnica y larga duración. En dos pacientes se registraron complicaciones pulmonares posquirúrgicas tempranas (embolia pulmonar), que provocaron su deceso, a pesar de recibir profilaxis y tratamiento. El paciente que en la evaluación inicial no mostró metástasis, evolucionó con lesiones pulmonares y hepáticas rápidamente progresivas, y 45 días después falleció.

En relación con los estudios histológicos, se observaron ductos neoplásicos, túbulos y papilas con

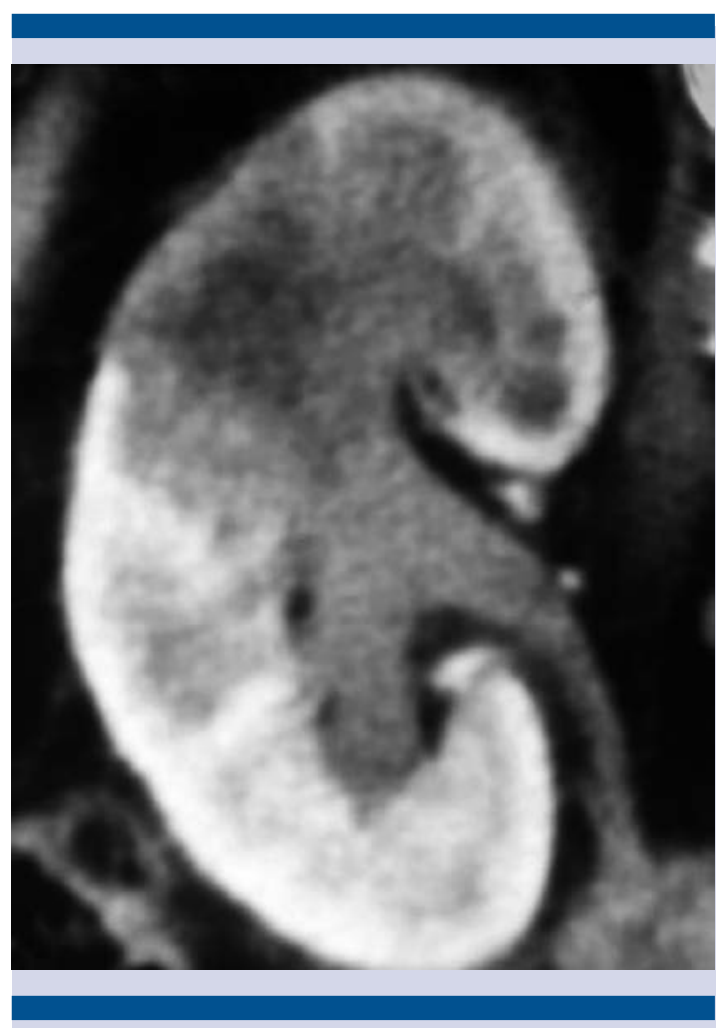

Figura 1. Tomografía en reconstrucción coronal. Se observa un tumor hipodenso en la parte central.

estroma desmoplásico e invasión al parénquima renal. En todos los casos se detectó invasión a la glándula suprarrenal. Los túbulos se encontraban cubiertos por células eosinofílicas con alto grado

Cuadro 1. Características clínicas y patológicas de los pacientes

\begin{tabular}{|c|c|c|c|c|c|c|c|c|c|c|}
\hline & Edad & $\begin{array}{c}\text { Tabaquismo } \\
\text { (paquetes/ } \\
\text { año) }\end{array}$ & Comorbilidades & $\begin{array}{l}\text { Síntomas } \\
\text { iniciales }\end{array}$ & ECOG & $\begin{array}{l}\text { Tamaño } \\
\text { del tumor } \\
\text { (TC) }\end{array}$ & $\begin{array}{l}\text { TNM } \\
\text { clínico }\end{array}$ & $\begin{array}{c}\text { Tamaño } \\
\text { del tumor } \\
\text { en } \\
\text { patología }\end{array}$ & $\begin{array}{l}\text { Inmunohis- } \\
\text { toquímica }\end{array}$ & $\begin{array}{c}\text { Supervi- } \\
\text { vencia } \\
\text { posne- } \\
\text { frectomía }\end{array}$ \\
\hline 1 & 65 & (5) & $\begin{array}{l}\text { Diabetes me- } \\
\text { Ilitus }\end{array}$ & $\begin{array}{c}\text { Dolor } \\
\text { abdominal, } \\
\text { disnea }\end{array}$ & 2 & $75 \mathrm{~mm}$ & $\begin{array}{c}\text { T2b N1 } \\
\text { M1 }\end{array}$ & $10 \mathrm{~cm}$ & $\begin{array}{c}\text { CK } 7+ \\
\text { Vimentina } \\
+, \text { CD } 34-\end{array}$ & 2 días \\
\hline 2 & 56 & $(25)$ & $\begin{array}{l}\text { Síndrome de } \\
\text { Dubin-Johnson }\end{array}$ & $\begin{array}{l}\text { Hematuria, } \\
\text { dolor abdo- } \\
\text { minal }\end{array}$ & 1 & $5.6 \mathrm{~mm}$ & $\begin{array}{c}\text { T1b N1 } \\
\text { M0 }\end{array}$ & $6.5 \mathrm{~cm}$ & $\begin{array}{l}\text { No reque- } \\
\text { rida }\end{array}$ & 45 días \\
\hline 3 & 61 & (2) & $\begin{array}{c}\text { Cáncer de } \\
\text { próstata }\end{array}$ & $\begin{array}{l}\text { Hematuria, } \\
\text { dolor abdo- } \\
\text { minal }\end{array}$ & 1 & $7.5 \mathrm{~mm}$ & $\begin{array}{c}\text { T2a N1 } \\
\text { M1 }\end{array}$ & $6 \mathrm{~cm}$ & $\begin{array}{c}\text { CK } 7+, \text { CD } \\
34-, \text { UEA- } \\
1+\end{array}$ & 13 días \\
\hline
\end{tabular}


nuclear (Figura 2). En dos pacientes se efectuó inmunohistoquímica para confirmar el diagnóstico, ambos resultaron positivos a citoqueratina 7(CK-7), 1 positivo a Ulex europeaus-1 (UEA-1) y ambos negativos a CD-34.

\section{DISCUSIÓN}

Los datos demográficos de los pacientes son similares a los expuestos en otras investigaciones. ${ }^{8}$ Anteriormente, el carcinoma de conductos
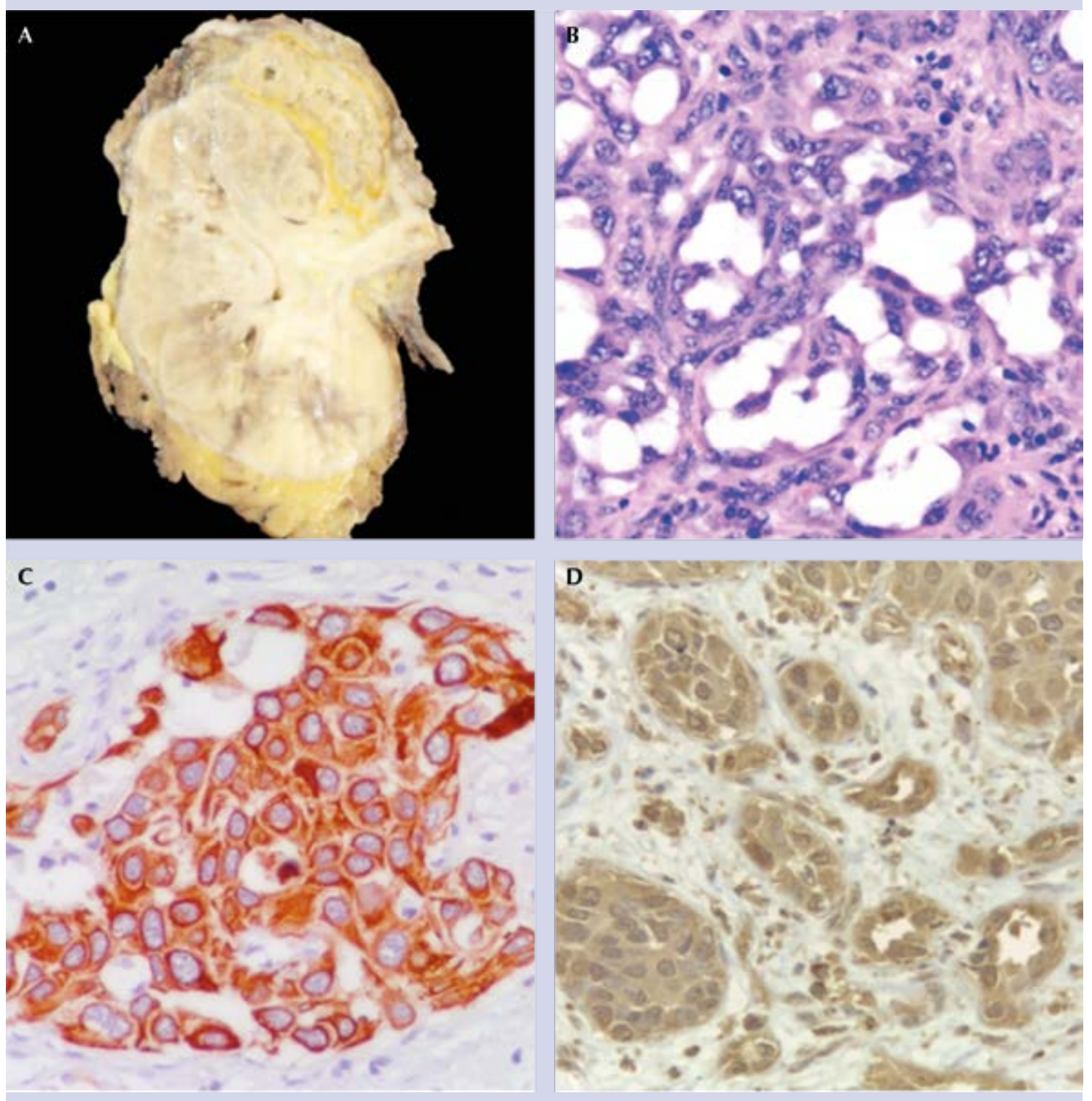

Figura 2. Estudio histopatológico. Imagen macroscópica del tumor (A), tinción con hematoxilina-eosina (B), Inmunohistoquímica positiva a CK-7 (C) y Ulex europeaus-1 (D). 
colectores fue descrito en sujetos con antecedentes familiares de neoplasias, pero sólo uno de nuestros casos tuvo antecedente familiar de primer grado con cáncer. Todos los pacientes tuvieron antecedente de tabaquismo de larga evolución, factor de riesgo conocido para padecer carcinoma de células claras, sin relación con carcinoma cromófobo, ${ }^{9}$ y que aún no ha sido evaluado en pacientes con carcinoma de conductos colectores.

La mayoría de los pacientes manifiesta síntomas al momento del diagnóstico. ${ }^{1}$ En nuestra serie, todos padecían dolor abdominal o lumbar y dos tuvieron hematuria. La evaluación radiográfica mostró las características de referencia descritas en este tipo de tumores.

Los tres pacientes cumplieron con los criterios de la $\mathrm{OMS}^{4}$ para establecer el diagnóstico de la enfermedad. Las determinaciones de UEA-1 y CK-7 representan marcadores confiables; sin embargo, no todos los carcinomas de conductos colectores resultan positivos al mismo procedimiento de inmunohistoquímica.

La invasión a la glándula suprarrenal ipsilateral es un factor relacionado con pobre pronóstico para carcinoma de células claras, ${ }^{10}$ pero hasta la fecha no ha sido evaluado en pacientes con carcinoma de conductos colectores.

La nefrectomía citorreductora es un procedimiento efectivo para prolongar la supervivencia en pacientes con carcinoma de células claras; sin embargo, en quienes padecen carcinoma de conductos colectores no ha mostrado beneficios y puede representar un factor de riesgo de morbilidad y mortalidad, como sucedió en este estudio, al registrar dos fallecimientos en el periodo postoperatorio temprano, debido a complicaciones pulmonares, similar a lo reportado por Majean y su grupo. ${ }^{3}$ Incluso puede indicar elevado riesgo de eventos tromboembólicos aso- ciados con la histología; por tanto, se requieren estudios adicionales de series más grandes. En casos similares proponemos, forzosamente, la prescripción de profilaxis antitrombótica y mantener un alto índice de sospecha para establecer el diagnóstico y tratamiento oportunos en sujetos con esta complicación.

Entre las múltiples opciones de terapia sistémica para pacientes con metástasis se encuentran la quimioterapia citotóxica e inmunoterapia. De acuerdo con diversos ensayos, la quimioterapia combinada con gemcitabina-cisplatino ha demostrado mayor efectividad, con tasa de reacción parcial de $26 \%{ }^{7}$ sin embargo, ninguno de nuestros pacientes tuvo supervivencia prolongada para comprobar el beneficio de este tratamiento.

\section{CONCLUSIONES}

El carcinoma de conductos colectores es una neoplasia poco estudiada. El pronóstico de los pacientes es desfavorable y hasta la fecha existen preguntas por responder, pues la mayor parte del conocimiento proviene de reportes de casos clínicos. Puesto que la nefrectomía citorreductora no proporciona beneficios en supervivencia y posiblemente se relaciona con morbilidad y mortalidad, y baja tasa de supervivencia, proponemos obtener una biopsia en pacientes con enfermedad localmente avanzada o metastásica y sugerentes de carcinoma de conductos colectores, con la finalidad de confirmar el diagnóstico mediante histología y evaluar el requerimiento de quimioterapia citotóxica.

\section{REFERENCIAS}

1. Kuroda N, Toi M, Hiroi M, et al. Review of collecting duct carcinoma with focus on clinical and pathobiological aspects. Histol Histopathol 2002;17:1329-1334.

2. Karakiewicz PI, Trinh QD, Rioux-Leclercq N, et al. Collecting duct renal cell carcinoma: a matched analysis of 41 cases. Eur Urol 2007;52:1140-1146. 
3. Méjean A, Rouprêt M, Larousserie F, et al. Is there a place for radical nephrectomy in the presence of metastatic collecting duct (Bellini) carcinoma? J Urol 2003;169:12871290.

4. Moch H, Humphrey PA, Ulbright TM, Reuter V. WHO classification of tumours of the urinary system and male genital organs. $4^{\mathrm{a}}$ ed. Lyon: International Agency for Research on Cancer, 2016.

5. Staehler M, Schöppler G, Haseke N, et al. Carcinoma of the collecting ducts of Bellini of the kidney: adjuvant chemotherapy followed by multikinase inhibition with sunitinib. Clin Genitourin Cancer 2009;7:58-61.

6. Dason S, Allard C, Sheridan-Jonah A, et al. Management of renal collecting duct carcinoma: a systematic review and the McMaster experience. Curr Oncol 2013;20:223-232.
7. Oudard S, Banu E, Vieillefond A, et al. Prospective multicenter phase ii study of gemcitabine plus platinum salt for metastatic collecting duct carcinoma: results of a GETUG (Groupe d'Etudes des Tumeurs Uro-Génitales) study. J Urol 2007;177:1698-1702.

8. Pepek JM, Johnstone P a S and Jani AB: Influence of demographic factors on outcome of collecting duct carcinoma: a Surveillance, Epidemiology, and End Results (SEER) database analysis. Clin Genitourin Cancer 2009;7:24-27.

9. Patel NH, Attwood KM, Hanzly M, et al. Comparative Analysis of Smoking as a Risk Factor among Renal Cell Carcinoma Histological Subtypes. J Urol 2015;194:640-646.

10. Alamdari FI, Ljungberg B. Adrenal metastasis in renal cell carcinoma: A recommendation for adjustment of the TNM staging system. Scand J Urol Nephrol 2005;39:277-282.

\section{AVISO IMPORTANTE}

La Revista Mexicana de Urología se convierte en una publicación solo digital (www.revistamexicanadeurologia.org.mx) con todas las ventajas que los medios electrónicos ofrecen.

Para consultar el texto completo de los artículos deberá registrarse por una sola vez con su correo electrónico, crear una contraseña, indicar su nombre, apellidos y especialidad.

Esta información es indispensable para saber qué consulta y cuáles son sus intereses, y poder en el futuro inmediato satisfacer sus necesidades de información. 